\title{
Cooperative Strategies for Relay-Aided Multi-Cell Wireless Networks with Backhaul
}

\author{
Jinfeng Du, Ming Xiao, and Mikael Skoglund \\ ACCESS Linnaeus Center, Royal Institute of Technology, Stockholm, Sweden \\ Email: jinfeng@kth.se, ming.xiao@ee.kth.se, mikael.skoglund@ee.kth.se
}

\begin{abstract}
We investigate cooperative strategies for relay-aided multi-source multi-destination wireless networks with backhaul support. Each source multicasts information to all destinations using a shared relay. We study cooperative strategies based on different network coding (NC) schemes, namely, finite field NC (FNC), linear NC (LNC), and lattice coding. To further exploit the backhaul connection, we also propose NC-based beam-forming (NBF). We measure the performance in term of achievable rates over Gaussian channels and observe significant gains over a benchmark scheme. The benefit of using backhaul is also clearly demonstrated in most of scenarios.
\end{abstract}

\section{INTRODUCTION}

Capacity bounds and various cooperative strategies for three-node relaying networks (source-relay-sink, or two cooperative sources and one sink) have been studied in [1], [2], where successive decoding, sliding-window forward decoding or backward decoding are used at the sink. The relay (or the other source) uses decode-and-forward (DF) or compress-andforward $(\mathrm{CF})$ to aid the transmission. Cooperative strategies and coding schemes are investigated for multiple-access relay channels (MARC) [3] involving multiple sources and a single destination, and for broadcast relay channels (BRC) [3], [4] where a single source transmits messages to multiple destinations. Recent results on capacity bounds for multiple-source multiple-destination relay networks, [5]-[7] and references therein, have provided valuable insights into the benefits of relaying, either half-duplex or full-duplex. Motivated by the MAC channel at the relay node where different messages add up by nature, various network coding (NC) approaches can be introduced to boost the sum rate. For instance, in a relay-aided two-source two-sink multicast network, achievable rates for a full-duplex amply-and-forward (AF) relay with analog NC have been studied in [6], and in [7] the relay uses lattice codes to do physical layer NC. Apart from introducing dedicated relay nodes to help the transmission, one can also utilize cooperative strategies among sources and/or among destinations [8], [9] through orthogonal conferencing channels.

In this paper, we aim at evaluating achievable sum-rate for various cooperative strategies when source cooperation and relaying are combined together. More specifically, we focus on a relay-aided two-source two-destination multicast network with backhaul support, as shown in Figure. 1. Sources $\mathcal{S}_{1}$ and $\mathcal{S}_{2}$ multicast their own information ( $W_{1}$ and $W_{2}$ respectively) to geographically separated destinations $\mathcal{D}_{1}$ and $\mathcal{D}_{2}$, with the help of a relay $\mathcal{R}$. This model arises from downlink wireless cellular networks where two base stations multicast to two mobile terminals, one in each cell, with the help of a dedicated relay deployed at the common cell boundary. Since the base

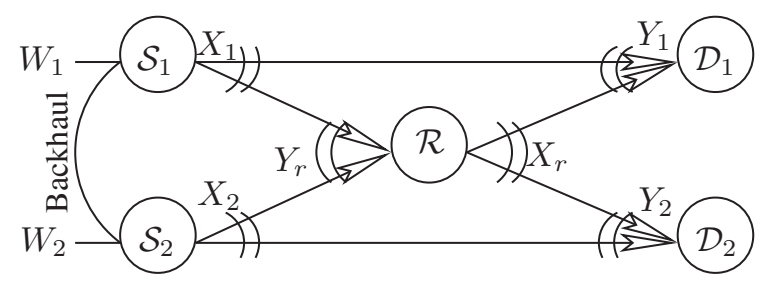

Figure 1. Two source nodes $\mathcal{S}_{1}$ and $\mathcal{S}_{2}$, connected with backhaul, multicast information $W_{1}$ and $W_{2}$ respectively to both destinations $\mathcal{D}_{1}$ and $\mathcal{D}_{2}$, with aid from a full-duplex relay node $\mathcal{R}$.

stations are connected through the (fiber or microwave) backhaul, more general network coding schemes can be used at the relay to cooperate with the sources' transmission. This model is interesting since it is a combination of relaying, MARC, $\mathrm{BRC}$, and sources cooperation. It can be extended to more general networks by tuning the channel gains within the range $[0, \infty)$. In this paper, we are interested in the scenario without cross channels between $\mathcal{S}_{1}$ and $\mathcal{D}_{2}$, or $\mathcal{S}_{2}$ and $\mathcal{D}_{1}$. In wireless cellular networks, such cross channels are normally too weak to be used or technically suppressed by the system. We restrict our analysis to fixed channel gains with a full-duplex DF relay. Extensions of cooperative strategies developed in this paper to general channel/relay setup are left to future work.

The rest of this paper is organized as follows. The system model is introduced in Section II. Various cooperative strategies are investigated in Section III and numerical results are presented in Section IV.

\section{SySTEM MODEL}

To simplify our analysis, we consider a symmetric channel gain scenario in Figure 1 (extension to non-symmetric channel gains is straightforward),

$$
\begin{aligned}
& Y_{1}^{(n)}=X_{1}^{(n)}+b X_{r}^{(n)}+Z_{1}^{(n)} \\
& Y_{2}^{(n)}=X_{2}^{(n)}+b X_{r}^{(n)}+Z_{2}^{(n)} \\
& Y_{r}^{(n)}=a X_{1}^{(n)}+a X_{2}^{(n)}+Z_{r}^{(n)}
\end{aligned}
$$

where $a \geq 0$ is the normalized channel gain for the sourcerelay links and $b \geq 0$ for the relay-destination links. $X_{i}^{(n)}$, $Y_{i}^{(n)}, Z_{i}^{(n)}, i=1,2, r$ are $n$-dimensional transmitted signals, received signals, and noise at nodes $\mathcal{S}_{1}, \mathcal{S}_{2}$ and $\mathcal{R}$, respectively. The noise components $Z_{i}(k), i=1,2, r$ and $k=1, \ldots, n$ are i.i.d. zero-mean unit-variance Gaussian random variables. Assuming perfect synchronization, $\mathcal{S}_{1}$ and $\mathcal{S}_{2}$ can cooperate with $\mathcal{R}$ and get coherent combining gains (i.e., beamforming) 
at the sinks, as stated in [1]-[3]. An average power constraint

$$
\frac{1}{n} \sum_{k=1}^{n} X_{i}^{2}(k) \leq P_{i}, \quad i=1,2, r,
$$

is assumed throughout this paper. We note that in practice, the backhaul has much higher capacity and lower error rates than the forward wireless channels. To ease our analysis and gain some insight on the original problem, in this paper the backhaul is assumed to be error-free and of unbounded capacity. This makes our system directly related to the MIMO relay channel scenario, as studied in e.g. [11]. However, we emphasize three main differences between the system model investigated in this paper and the scenario with a single source equipped with two transmit antennas: Each source/antenna is subject to individual power constraint; the DF relay combines messages from each source by performing $\mathrm{NC}$ rather than forwarding them separately through orthogonal channels; cooperative strategies proposed for this model can be directly extended to the finite-rate backhual scenario with the help of superposition coding or time-sharing strategies. However, the capacity upper bounds derived for the MIMO relay channel, e.g., Theorem 3.1 in [11] which motivated by the cut-set bound, are still relevant here.

\section{CoOperative STRATEGIES}

Source $\mathcal{S}_{i}, i=1,2$, similar to [1]-[3], [5], divides its messages $W_{i}$ into $B$ blocks $W_{i, 1}, W_{i, 2}, \ldots, W_{i, B}$ with $k_{i}$ bits each. The transmission is completed over $B+1$ blocks. At the first block the two sources exchange $W_{i, 1}$ over the backhaul and meanwhile broadcast them over the relay channels; in block $t$, source $\mathcal{S}_{i}$ exchanges $W_{i, t}$ through the backhual and broadcasts its codeword $X_{i, t}^{(n)}$, which is a function of $\left(W_{i, t}, W_{1, t-1}, W_{2, t-1}\right)$, over the relay channels; in block $B+1$ only $W_{i, B}$ is broadcasted. As each transmission is over $n$ channel uses, and assuming that using the backhual is free, the overall rate is $\frac{B k_{i}}{(B+1) n}$ bits per channel use, which converges to $R_{i}=\frac{k_{i}}{n}$ when $B$ goes to infinity. In what follows, we shall investigate the achievable rates for different cooperative strategies with network coding and backhaul.

\section{A. Finite-field Network Coding with DF $(D F+F N C)$}

After receiving $Y_{r, t-1}^{(n)}, \mathcal{R}$ decodes $\left(\hat{W}_{1, t-1}, \hat{W}_{2, t-1}\right)$ jointly by maximum-likelihood or typical sequence decoding, and forms network coded information $W_{r, t}=\hat{W}_{1, t-1} \oplus \hat{W}_{2, t-1}$ (in $\mathrm{GF}(2))$. If the lengths of $W_{1, t-1}$ and $W_{2, t-1}$ are not equal, i.e., $R_{1} \neq R_{2}$, we can append zeros at the end of the shorter message. Then at block $t, \mathcal{R}$ picks up a codeword ${ }^{1} U^{(n)}\left(W_{r, t}\right)$ corresponding to $W_{r, t}$ from a codebook and transmits

$$
X_{r, t}^{(n)}=\sqrt{P_{r}} U^{(n)}\left(W_{r, t}\right) .
$$

$\mathcal{S}_{1}$ and $\mathcal{S}_{2}$, exchanging $W_{1, t-1}$ and $W_{2, t-1}$ via the backhaul in block $t-1$, knows $W_{r, t}$ if decoding at $\mathcal{R}$ is reliable. By cooperating with $\mathcal{R}, \mathcal{S}_{1}$ and $\mathcal{S}_{2}$ at block $t$ transmit

$X_{1, t}^{(n)}=\sqrt{\alpha_{1} P_{1}} V_{1}^{(n)}\left(W_{1, t}\right)+\sqrt{\left(1-\alpha_{1}\right) P_{1}} U^{(n)}\left(W_{r, t}\right)$,
$X_{2, t}^{(n)}=\sqrt{\alpha_{2} P_{2}} V_{2}^{(n)}\left(W_{2, t}\right)+\sqrt{\left(1-\alpha_{2}\right) P_{2}} U^{(n)}\left(W_{r, t}\right)$,

${ }^{1}$ We relate the message to its codeword in the way of an encoder function, and their dependence will be omitted where appropriate. Each codeword is generated in the usual memoryless fashion. where $0 \leq \alpha_{1}, \alpha_{2} \leq 1$ are power allocation parameters for cooperation. $V_{1}^{(n)}, V_{2}^{(n)}$ are codewords from independent codebooks. $\mathcal{S}_{1}$ and $\mathcal{S}_{2}$ synchronize their transmission with $\mathcal{R}$ so that $X_{i}^{(n)}$ (More precisely, only the part of relaying signal $\left.U^{(n)}\right)$ can be coherently added with $X_{r}^{(n)}$, i.e.,

$Y_{1, t}^{(n)}=\sqrt{\alpha_{1} P_{1}} V_{1}^{(n)}+\left(\sqrt{\left(1-\alpha_{1}\right) P_{1}}+b \sqrt{P_{r}}\right) U^{(n)}+Z_{1}^{(n)}$,

$Y_{2, t}^{(n)}=\sqrt{\alpha_{2} P_{2}} V_{2}^{(n)}+\left(\sqrt{\left(1-\alpha_{2}\right) P_{2}}+b \sqrt{P_{r}}\right) U^{(n)}+Z_{2}^{(n)}$,

$$
\begin{aligned}
Y_{r, t}^{(n)}= & a\left(\sqrt{\left(1-\alpha_{1}\right) P_{1}}+\sqrt{\left(1-\alpha_{2}\right) P_{2}}\right) U^{(n)}+Z_{r}^{(n)} \\
& +a \sqrt{\alpha_{1} P_{1}} V_{1}^{(n)}+a \sqrt{\alpha_{2} P_{2}} V_{2}^{(n)} .
\end{aligned}
$$

Assuming $\hat{W}_{1, t-1}$ was successfully decoded by $\mathcal{D}_{1}$, at the end of block $t, \mathcal{D}_{1}$ recovers $\left(\hat{W}_{r, t}, \hat{W}_{1, t}\right)$ jointly from $Y_{1, t}^{(n)}$, and then decodes $\hat{W}_{2, t-1}=\hat{W}_{r, t} \oplus \hat{W}_{1, t-1}$, and similarly for $\mathcal{D}_{2}$. The relay $\mathcal{R}$ detects jointly $\left(\hat{W}_{1, t}, \hat{W}_{2, t}\right)$ from $(5 \mathrm{c})$ by first cancelling out $U^{(n)}$. Since the channel is additive memoryless Gaussian, there exist joint Gaussian variables $\left(V_{1}^{(n)}, V_{2}^{(n)}, U^{(n)}\right)$ such that the mutual information between channel input and output can be maximized, similar to [7]. The achievable rate region is therefore obtained as

$$
\begin{aligned}
& R_{1}<\min \left\{C\left(a^{2} \alpha_{1} P_{1}\right), C\left(\alpha_{1} P_{1}\right), C\left(\left(\sqrt{\left(1-\alpha_{2}\right) P_{2}}+b \sqrt{P_{r}}\right)^{2}\right)\right\}, \\
& R_{2}<\min \left\{C\left(a^{2} \alpha_{2} P_{2}\right), C\left(\alpha_{2} P_{2}\right), C\left(\left(\sqrt{\left(1-\alpha_{1}\right) P_{1}}+b \sqrt{P_{r}}\right)^{2}\right)\right\}, \\
& R_{1}+R_{2}<\min \left\{C\left(P_{1}+b^{2} P_{r}+2 b \sqrt{\left(1-\alpha_{1}\right) P_{1} P_{r}}\right),\right. \\
& \left.C\left(a^{2}\left(\alpha_{1} P_{1}+\alpha_{2} P_{2}\right)\right), C\left(P_{2}+b^{2} P_{r}+2 b \sqrt{\left(1-\alpha_{2}\right) P_{2} P_{r}}\right)\right\},
\end{aligned}
$$

with the union taken over $0 \leq \alpha_{1}, \alpha_{2} \leq 1$. Here, $C(x)=$ $\frac{1}{2} \log _{2}(1+x)$ is the Gaussian capacity function. The constraints in $R_{1}$ correspond to the conditions that $W_{1}$ can be decoded reliably at $\mathcal{R}$, at $\mathcal{D}_{1}$, and that the network coded information $W_{r}$ can be decoded at $\mathcal{D}_{2}$. It is similar for $R_{2}$. The constraints in $R_{1}+R_{2}$ refer to successful decoding at $\mathcal{D}_{1}, \mathcal{R}$, and $\mathcal{D}_{2}$, respectively.

For the symmetric scenario with $P_{1}=P_{2}=P_{r}=P$ and $R_{1}=R_{2}=R$, by setting $\alpha_{1}=\alpha_{2}=\alpha$, (6) becomes

$$
\begin{aligned}
R<\max _{0 \leq \alpha \leq 1} \min \{ & \frac{1}{2} \log _{2}(1+\alpha P), \frac{1}{4} \log _{2}\left(1+2 a^{2} P \alpha\right), \\
& \left.\frac{1}{4} \log _{2}\left(1+\left(1+b^{2}+2 b \sqrt{1-\alpha}\right) P\right)\right\} .
\end{aligned}
$$

Without backhual, $\mathcal{S}_{1}$ and $\mathcal{S}_{2}$ cannot know/estimate $W_{r}$ and therefore cannot cooperate with $\mathcal{R}$, i.e. $\alpha_{1}=\alpha_{2}=1$. Hence, no coherent combining gain can be achieved.

\section{B. Linear Network Coding with DF $(D F+L N C)$}

Similar to the cooperative strategies with a DF relay discussed in [3] for the MARC and in [7] for multiple multicast, two sources cooperate in their transmission with $\mathcal{R}$. When linear network coding (LNC) is used in the signal domain, $\mathcal{R}$ essentially performs superposition coding. As in Sec. III-A, $\mathcal{R}$ decodes $\left(\hat{W}_{1, t-1}, \hat{W}_{2, t-1}\right)$ at the end of block $t-1$, and then at block $t$ it transmits

$X_{r, t}^{(n)}=\sqrt{\alpha_{r} P_{r}} U_{1}^{(n)}\left(\hat{W}_{1, t-1}\right)+\sqrt{\left(1-\alpha_{r}\right) P_{r}} U_{2}^{(n)}\left(\hat{W}_{2, t-1}\right)$, 
where $U_{1}^{(n)}\left(\hat{W}_{1, t-1}\right)$ and $U_{2}^{(n)}\left(\hat{W}_{2, t-1}\right)$ are codewords from independent codebooks and $0 \leq \alpha_{r} \leq 1$ is the power allocation parameter. Meanwhile $\mathcal{S}_{1}$ and $\mathcal{S}_{2}$ cooperate their transmission with $\mathcal{R}$ and transmit

$X_{1, t}^{(n)}=\sqrt{\alpha_{1}^{\prime} P_{1}} U_{1}^{(n)}+\sqrt{\alpha_{1}^{\prime \prime} P_{1}} U_{2}^{(n)}+\sqrt{\left(1-\alpha_{1}^{\prime}-\alpha_{1}^{\prime \prime}\right) P_{1}} V_{1}^{(n)}$,
$X_{2, t}^{(n)}=\sqrt{\alpha_{2}^{\prime} P_{2}} U_{2}^{(n)}+\sqrt{\alpha_{2}^{\prime \prime} P_{2}} U_{1}^{(n)}+\sqrt{\left(1-\alpha_{2}^{\prime}-\alpha_{2}^{\prime \prime}\right) P_{2}} V_{2}^{(n)}$,

where $0 \leq \alpha_{i}^{\prime}, \alpha_{i}^{\prime \prime} \leq 1, i=1,2$, are power allocation parameters. $V_{1}^{(n)}\left(W_{1, t}, W_{1, t-1}\right)$ and $V_{2}^{(n)}\left(W_{2, t}, W_{2, t-1}\right)$ are codewords from independent codebooks.

At the end of block $t, \mathcal{R}$ uses the forward decoding technique [3] and decodes $\left(W_{1, t}, W_{2, t}\right)$ reliably from $Y_{r, t}^{(n)}$ if $n$ is large, its past detection is correct, and

$$
\begin{array}{r}
n R_{1} \leq I\left(X_{1}^{(n)} ; Y_{r}^{(n)} \mid U_{1}^{(n)}, U_{2}^{(n)}, X_{2}^{(n)}, X_{r}^{(n)}\right), \\
n R_{2} \leq I\left(X_{2}^{(n)} ; Y_{r}^{(n)} \mid U_{1}^{(n)}, U_{2}^{(n)}, X_{1}^{(n)}, X_{r}^{(n)}\right), \\
n\left(R_{1}+R_{2}\right) \leq I\left(X_{1}^{(n)}, X_{2}^{(n)} ; Y_{r}^{(n)} \mid U_{1}^{(n)}, U_{2}^{(n)}, X_{r}^{(n)}\right),
\end{array}
$$

where $X_{1}, X_{2}, X_{r}$ are independent given $\left(U_{1}, U_{2}\right)$. At $\mathcal{D}_{1}$ and $\mathcal{D}_{2}$, on the other hand, we use backward decoding as follows. At block $B+1$, no new message is transmitted and the received signal at $\mathcal{D}_{1}\left(\mathcal{D}_{2}\right)$ only depends on $\left(W_{1, B}, W_{2, B}\right)$, which can be decoded reliably if

$n R_{1} \leq \min \left\{I\left(X_{1}^{(n)}, X_{r}^{(n)} ; Y_{1}^{(n)} \mid U_{2}^{(n)}\right), I\left(X_{r}^{(n)} ; Y_{2}^{(n)} \mid U_{2}^{(n)}, X_{2}^{(n)}\right)\right\}$, $n R_{2} \leq \min \left\{I\left(X_{2}^{(n)}, X_{r}^{(n)} ; Y_{2}^{(n)} \mid U_{1}^{(n)}\right), I\left(X_{r}^{(n)} ; Y_{1}^{(n)} \mid U_{1}^{(n)}, X_{1}^{(n)}\right)\right\}$ $n\left(R_{1}+R_{2}\right) \leq \min \left\{I\left(X_{1}^{(n)}, X_{r}^{(n)} ; Y_{1}^{(n)}\right), I\left(X_{2}^{(n)}, X_{r}^{(n)} ; Y_{2}^{(n)}\right)\right\}$,

are satisfied. After decoding $\left(W_{1, B}, W_{2, B}\right)$ successfully, only $W_{1, B-1}$ and $W_{2, B-1}$ are unknown in $Y_{1, B}^{(n)}$ and $Y_{2, B}^{(n)}$, and we can repeat this process backwards until all the messages are recovered. Applying the Gaussian conditions in (8) and (9), after some algebra the achievable rate region can be found as

$$
\begin{array}{r}
R_{1}<\min \left\{C\left(\left(1-\alpha_{1}^{\prime}-\alpha_{1}^{\prime \prime}\right) P_{1}+\left[\sqrt{\alpha_{1}^{\prime} P_{1}}+b \sqrt{\alpha_{r} P_{r}}\right]^{2}\right),\right. \\
\left.C\left(a^{2} P_{1}\left(1-\alpha_{1}^{\prime}-\alpha_{1}^{\prime \prime}\right)\right), C\left(\left[\sqrt{\alpha_{2}^{\prime \prime} P_{2}}+b \sqrt{\alpha_{r} P_{r}}\right]^{2}\right)\right\},
\end{array}
$$

$$
\begin{array}{r}
R_{2}<\min \left\{C\left(\left(1-\alpha_{2}^{\prime}-\alpha_{2}^{\prime \prime}\right) P_{2}+\left[\sqrt{\alpha_{2}^{\prime} P_{2}}+b \sqrt{\left(1-\alpha_{r}\right) P_{r}}\right]^{2}\right),\right. \\
\left.C\left(a^{2} P_{2}\left(1-\alpha_{2}^{\prime}-\alpha_{2}^{\prime \prime}\right)\right), C\left(\left[\sqrt{\alpha_{1}^{\prime \prime} P_{1}}+b \sqrt{\left(1-\alpha_{r}\right) P_{r}}\right]^{2}\right)\right\}, \\
R_{1}+R_{2}<\min \left\{C\left(a^{2}\left(1-\alpha_{1}^{\prime}-\alpha_{1}^{\prime \prime}\right) P_{1}+a^{2}\left(1-\alpha_{2}^{\prime}-\alpha_{2}^{\prime \prime}\right) P_{2}\right),\right. \\
\quad C\left(P_{1}+b^{2} P_{r}+2 b \sqrt{P_{1} P_{r}}\left[\sqrt{\alpha_{1}^{\prime} \alpha_{r}}+\sqrt{\alpha_{1}^{\prime \prime}\left(1-\alpha_{r}\right)}\right]\right), \\
\left.C\left(P_{2}+b^{2} P_{r}+2 b \sqrt{P_{2} P_{r}}\left[\sqrt{\alpha_{2}^{\prime \prime} \alpha_{r}}+\sqrt{\alpha_{2}^{\prime}\left(1-\alpha_{r}\right)}\right]\right)\right\},
\end{array}
$$

with the union taken over $0 \leq \alpha_{r}, \alpha_{1}^{\prime}, \alpha_{1}^{\prime \prime}, \alpha_{2}^{\prime}, \alpha_{2}^{\prime \prime} \leq 1$, $\alpha_{1}^{\prime}+\alpha_{1}^{\prime \prime} \leq 1, \alpha_{2}^{\prime}+\alpha_{2}^{\prime \prime} \leq 1$. The constraints in $R_{1}$ refer to the condition that $W_{1}$ can be decoded at $\mathcal{D}_{1}, \mathcal{R}$, and $\mathcal{D}_{2}$, respectively, and similarly for $R_{2}$. For $R_{1}+R_{2}$, the three terms correspond to the constraint that $\left(W_{1}, W_{2}\right)$ can be jointly decoded at $\mathcal{R}, \mathcal{D}_{1}$, and $\mathcal{D}_{2}$, respectively.
For the symmetric scenario, by setting $\alpha_{1}^{\prime}=\alpha_{2}^{\prime}=\alpha^{\prime}$, $\alpha_{1}^{\prime \prime}=\alpha_{2}^{\prime \prime}=\alpha^{\prime \prime}$, and $\alpha_{r}=1 / 2$, (10) can be translated to the following equal rate constraint

$$
\begin{array}{r}
R<\max _{\substack{\alpha^{\prime} \geq 0, \alpha^{\prime \prime} \geq 0 \\
0 \leq \alpha^{\prime}+\alpha^{\prime \prime} \leq 1}} \min \left\{C\left(\left[1-\alpha^{\prime \prime}+b^{2} / 2+b \sqrt{2 \alpha^{\prime}}\right] P\right),\right. \\
\frac{1}{2} C\left(2 a^{2} P\left(1-\alpha^{\prime}-\alpha^{\prime \prime}\right)\right), C\left(\left[\alpha^{\prime \prime}+b^{2} / 2+b \sqrt{2 \alpha^{\prime \prime}}\right] P\right), \\
\frac{1}{2} C\left(\left[1+b^{2}+b \sqrt{2 \alpha^{\prime}}+b \sqrt{2 \alpha^{\prime \prime}}\right] P\right) .
\end{array}
$$

Without the backhaul, the relaying signal is only partially known by the source nodes, i.e., setting $\alpha_{1}^{\prime \prime}=\alpha_{2}^{\prime \prime}=0$ instead.

\section{Physical Layer Network Coding by Lattice Coding}

In contrast to Sec. III-A where $\mathcal{R}$ first decodes $\left(W_{1}, W_{2}\right)$ and then generates a $\mathrm{NC}$ message $W_{r}$, the relay can directly decode the NC message from $Y_{r}^{(n)}$ by using a nested lattice encoding and decoding strategy, as stated in [7], [10]. Without loss of generality, we assume that $P_{1} \leq P_{2}$ (hence $R_{1} \leq R_{2}$ ). $\mathcal{S}_{1}$ encodes $W_{1, b}$ via a nested lattice code with power $0 \leq \delta \leq$ $P_{1}$ and cooperates with the relaying signal with the rest power. $\mathcal{S}_{2}$ splits its message $W_{2, b}$ into two parts $\left[W_{2, b}^{\prime}, W_{2, b}^{\prime \prime}\right]$, where $W_{2, b}^{\prime}$ has the same length as $W_{1, b}$, and encodes $W_{2, b}^{\prime}$ with power $\delta$ using the same nested lattice code, and encodes $W_{2, b}^{\prime \prime}$ using a random code with power $0 \leq \epsilon \leq P_{2}-\delta$. The rest power of $\mathcal{S}_{2}$ is used to cooperate with the relay. The following rate region can be proved (proof is omitted here due to space limitation) to be achievable by using lattice code

$$
\begin{gathered}
R_{1}<\min \left\{C\left(-\frac{1}{2}+a^{2} \delta\right), C(\delta), C\left(\left(\sqrt{P_{2}-\delta-\epsilon}+b \sqrt{P_{r}}\right)^{2}\right)\right\}, \\
R_{2}<\min \left\{C\left(-\frac{1}{2}+a^{2} \delta+a^{2} \epsilon / 2\right), C(\delta+\epsilon),\right. \\
\left.C\left(\left(\sqrt{P_{1}-\delta}+b \sqrt{P_{r}}\right)^{2}\right)\right\}, \quad(11) \\
R_{1}+R_{2}<\min \left\{C\left(\delta+\left(\sqrt{P_{1}-\delta}+b \sqrt{P_{r}}\right)^{2}\right),\right. \\
\left.C\left(\delta+\epsilon+\left(\sqrt{P_{2}-\delta-\epsilon}+b \sqrt{P_{r}}\right)^{2}\right)\right\},
\end{gathered}
$$

with the union taken over $0 \leq \delta \leq P_{1}$ and $0 \leq \epsilon \leq P_{2}-\delta$. The first term in $R_{1}\left(R_{2}\right)$ comes from the constraint at $\mathcal{R}$ when using a nested lattice encoding and decoding strategy. For the symmetric scenario, by setting $\epsilon=0, \delta=P \alpha$ we get

$$
\begin{array}{r}
R<\max _{0 \leq \alpha \leq 1} \min \left\{C(\alpha P), C\left(-\frac{1}{2}+a^{2} P \alpha\right),\right. \\
\left.C\left((b+\sqrt{1-\alpha})^{2} P\right), \frac{1}{2} C\left(\left(1+b^{2}+2 b \sqrt{1-\alpha}\right) P\right)\right\} .
\end{array}
$$

Without the backhual, the network coded message is not known by the source nodes, i.e., $\delta=P_{1}$ and $\epsilon=P_{2}-P_{1}$.

\section{Network Coding Based Beam-forming with $D F(D F+N B F)$}

We propose a new strategy performing network coding at $\mathcal{S}_{1}$ and $\mathcal{S}_{2}$ rather than $\mathcal{R}$, requiring $B+2$ blocks in total: $\left(W_{1, t-1}, W_{2, t-1}\right)$ are exchanged via the backhaul during block $t-1$; at block $t$ they are network coded into $W_{t}$ and transmitted; at block $t+1, \hat{W}_{t}$ is transmitted by $\mathcal{R}$. At block $t$, the transmitted signals over the relay channels are

$X_{r, t}^{(n)}=\sqrt{P_{r}} U^{(n)}\left(\hat{W}_{t-1}\right)$

$X_{i, t}^{(n)}=\sqrt{\alpha_{i} P_{i}} V^{(n)}\left(W_{t}, W_{t-1}\right)+\sqrt{\left(1-\alpha_{i}\right) P_{i}} U^{(n)}, i=1,2$. 
$\mathcal{S}_{1}$ and $\mathcal{S}_{2}$ synchronize their transmission with $\mathcal{R}$ like beamforming so that the received signals can coherently add up at both $\mathcal{D}_{1}$ and $\mathcal{D}_{2}$, but not at $\mathcal{R}$ due to wave propagation [12]. By performing forward decoding at the relay and backward decoding at destinations, the achievable rate region becomes

$$
\begin{aligned}
& R_{1}+R_{2}<\min \left\{C\left(P_{1}+b^{2} P_{r}+2 b \sqrt{\left(1-\alpha_{1}\right) P_{1} P_{r}}\right),\right. \\
& \left.C\left(a^{2}\left(\alpha_{1} P_{1}+\alpha_{2} P_{2}\right)\right), C\left(P_{2}+b^{2} P_{r}+2 b \sqrt{\left(1-\alpha_{2}\right) P_{2} P_{r}}\right)\right\},
\end{aligned}
$$

with the union taken over the power allocation parameters $0 \leq \alpha_{1}, \alpha_{2} \leq 1$. The terms in (14) indicate the constraint at $\mathcal{D}_{1}, \mathcal{R}$, and $\mathcal{D}_{2}$, respectively.

For the symmetric scenario, by setting $\alpha_{1}=\alpha_{2}=\alpha$ in (14) we get

$R<\max _{0 \leq \alpha \leq 1} \min \left\{\frac{1}{2} C\left(2 a^{2} P \alpha\right), \frac{1}{2} C\left(\left(1+b^{2}+2 b \sqrt{1-\alpha}\right) P\right)\right\}$.

Without the backhual, this strategy is impossible.

\section{E. Time Sharing Relay with $D F(D F+T D)$}

To illustrate the gain of network coding, we consider a cooperative strategy where the relay helps two sources alternatively, i.e., $\mathcal{R}$ is time shared by $\mathcal{S}_{1}$ and $\mathcal{S}_{2}$. In contrast to the orthogonal scheme described in Sec. IV of [5] for the interference relay channel without backhaul, $\mathcal{S}_{1}$ and $\mathcal{S}_{2}$ in our scheme cooperate via $\mathcal{R}$. During block $t, W_{1, t}$ and $W_{2, t}$ are exchanged via the backhaul and the transmission over the relay channels is divided into two parts. During the first part of block $t$, the transmitted signals are

$X_{1, t_{1}}^{(n)}=\sqrt{\frac{\alpha_{1} P_{1}}{\beta}} V_{1}^{(n)}\left(W_{1, t}, W_{2, t-1}\right)+\sqrt{\frac{\left(1-\alpha_{1}\right) P_{1}}{\beta}} U_{2}^{(n)}\left(W_{2, t-1}\right)$, $X_{r, t_{1}}^{(n)}=\sqrt{P_{r}} U_{2}^{(n)}\left(W_{2, t-1}\right), \quad X_{2, t_{1}}^{(n)}=0$,

where $0 \leq \alpha_{1} \leq 1$ is the power allocation parameter and $0 \leq \beta \leq 1$ is the time sharing parameter. Note that the transmission power $P_{1} / \beta$ is used in $X_{1, t_{1}}^{(n)}$ to meet the average power constraint (2). The relay decodes $W_{1, t}$ given $W_{2, t-1}$ and then encodes it to $U_{1}^{(n)}\left(W_{1, t}\right)$. Similarly, during the remaining part of block $t$, the transmitted signals are

$$
\begin{aligned}
& X_{2, t_{2}}^{(n)}=\sqrt{\frac{\alpha_{2} P_{2}}{1-\beta}} V_{2}^{(n)}\left(W_{2, t}, W_{1, t}\right)+\sqrt{\frac{\left(1-\alpha_{2}\right) P_{2}}{1-\beta}} U_{1}^{(n)}\left(W_{1, t}\right), \\
& X_{r, t_{2}}^{(n)}=\sqrt{P_{r}} U_{1}^{(n)}\left(W_{1, t}\right), \quad X_{1, t_{2}}^{(n)}=0 .
\end{aligned}
$$

At the end of block $t, \mathcal{R}$ decodes $W_{2, t}$ given $W_{1, t}$, and $\mathcal{D}_{1}$ can retrieve $\left(W_{1, t}, W_{2, t-1}\right)$ reliably using sliding-window decoding based on the received signals during block $t$. Similarly, after the first part of block $t+1, \mathcal{D}_{2}$ can decode $\left(W_{2, t}, W_{1, t}\right)$ reliably based on signals received from the first part of block $t+1$ and the second part of block $t$. The achievable rate region is therefore defined by

$$
\begin{gathered}
R_{1}<\min \left\{\beta C\left(\alpha_{1} a^{2} P_{1} / \beta\right), \beta C\left(\alpha_{1} P_{1} / \beta\right)+(1-\beta) C\left(b^{2} P_{r}\right),\right. \\
\left.(1-\beta) C\left(b^{2} P_{r}+P_{2} /(1-\beta)+2 b \sqrt{\left(1-\alpha_{2}\right) P_{2} P_{r} /(1-\beta)}\right)\right\},
\end{gathered}
$$

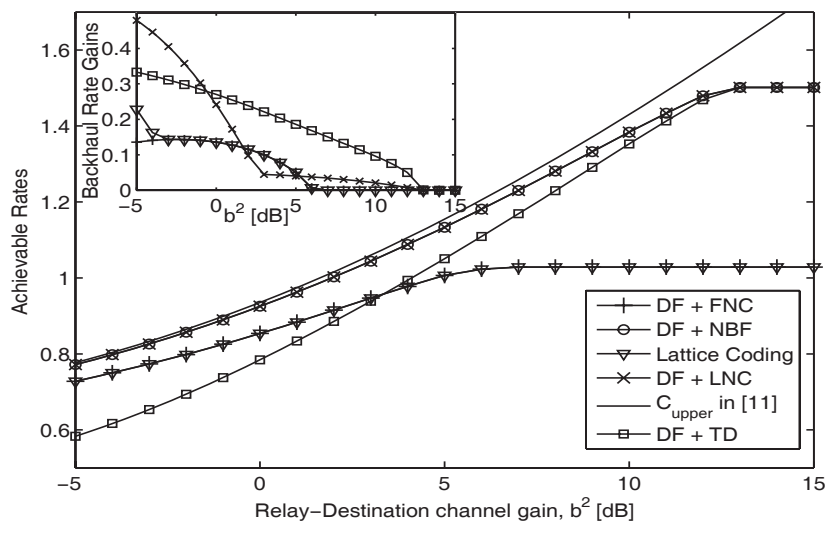

Figure 2. Effects of the relay-destination channel gain $b^{2}$ on the achievable rates with backhaul, when $P / \sigma^{2}=5 \mathrm{~dB}$ and the source-relay channel gain $a^{2}=10 \mathrm{~dB}$. The rate gains compared to the schemes without backhaul are also presented in the sub-figure.

$$
\begin{gathered}
R_{2}<\min \left\{(1-\beta) C\left(\frac{\alpha_{2} a^{2} P_{2}}{1-\beta}\right),(1-\beta) C\left(\frac{\alpha_{2} P_{2}}{1-\beta}\right)+\beta C\left(b^{2} P_{r}\right),\right. \\
\left.\beta C\left(b^{2} P_{r}+P_{1} / \beta+2 b \sqrt{\left(1-\alpha_{1}\right) P_{1} P_{r} / \beta}\right)\right\}, \\
R_{1}+R_{2}<\min \left\{(1-\beta) C\left(b^{2} P_{r}\right)\right. \\
+\beta C\left(b^{2} P_{r}+P_{1} / \beta+2 b \sqrt{\left(1-\alpha_{1}\right) P_{1} P_{r} / \beta}\right), \\
\left.\beta C\left(b^{2} P_{r}\right)+(1-\beta) C\left(b^{2} P_{r}+\frac{P_{2}}{1-\beta}+2 b \sqrt{\frac{\left(1-\alpha_{2}\right) P_{2} P_{r}}{1-\beta}}\right)\right\},
\end{gathered}
$$

with the union taken over all $0 \leq \alpha_{1}, \alpha_{2} \leq 1$ and $0 \leq \beta \leq 1$. The three constraints in $R_{1}\left(R_{2}\right)$ correspond to the condition that $W_{1, t}\left(W_{2, t}\right)$ is able to be decoded at the relay, $\mathcal{D}_{1}\left(\mathcal{D}_{2}\right)$, and $\mathcal{D}_{2}\left(\mathcal{D}_{1}\right)$ respectively. The first term of $R_{1}+R_{2}$ in (16) is the decoding constraint at $\mathcal{D}_{1}$ and the second term refers to successful decoding at $\mathcal{D}_{2}$.

By setting $\alpha_{1}=\alpha_{2}=\alpha$ and $\beta=\frac{1}{2}$, (16) becomes

$$
\begin{gathered}
R<\max _{0 \leq \alpha \leq 1} \min \left\{\frac{1}{4} \log _{2}\left(1+P\left(2+b^{2}+2 b \sqrt{2-2 \alpha}\right)\right),\right. \\
\frac{1}{4} \log _{2}\left(1+2 \alpha P+b^{2} P+2 \alpha b^{2} P^{2}\right), \frac{1}{4} \log _{2}\left(1+2 a^{2} P \alpha\right), \\
\left.\frac{1}{8} \log _{2}\left(1+b^{2} P\right)\left(1+P\left(2+b^{2}+2 b \sqrt{2-2 \alpha}\right)\right)\right\} .
\end{gathered}
$$

Without backhaul, sources can only encode over their own messages. Therefore we have $\alpha_{1}=\alpha_{2}=1$ and the first term in (17) reduces to $\frac{1}{4} \log _{2}\left(1+b^{2} P\right)$.

\section{NumericAl Results}

We focus on the symmetric scenario and compare the achievable equal rate $R$, and demonstrate rate gains by using network coding and utilizing the backhaul. The capacity upper bound derived in [11], Theorem 3.1, by setting $M_{1}=2$ and $M_{2}=1$ and modifying the channel matrices accordingly, serves as a baseline to evaluate our strategies.

In Fig. 2, we investigate the impact of the relay-destination link quality $b^{2}$ on the achievable rates for different cooperative strategies, with fixed source-relay channel gain $a^{2}=10 \mathrm{~dB}$ and the transmit power $P / \sigma^{2}=5 \mathrm{~dB}$. With backhaul, substantial rate gains can be achieved by performing LNC or NBF compared to the time sharing relay. Network coding in the finite field or by using lattice code is preferred for small $b^{2}$. 


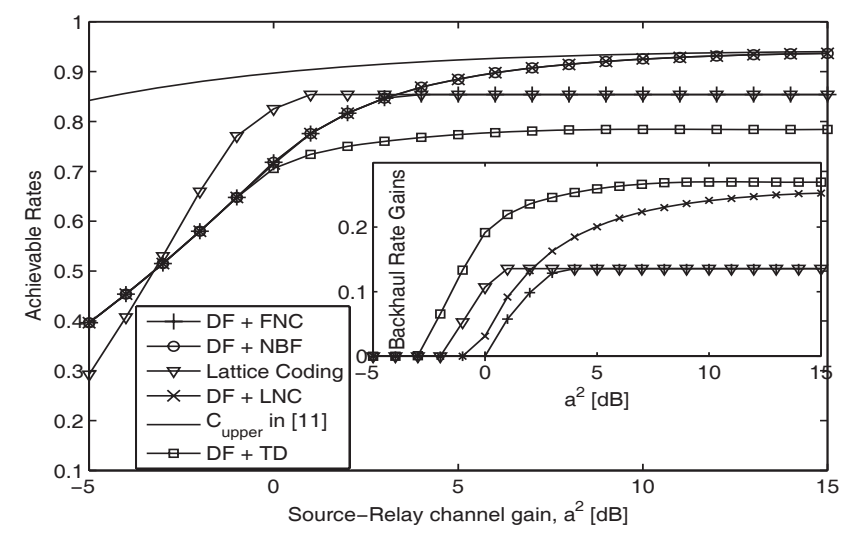

Figure 3. Effects of the source-relay channel gain $a^{2}$ on the achievable rates with $P / \sigma^{2}=5 \mathrm{~dB}$ and the relay-destination channel gain $b^{2}=0 \mathrm{~dB}$.

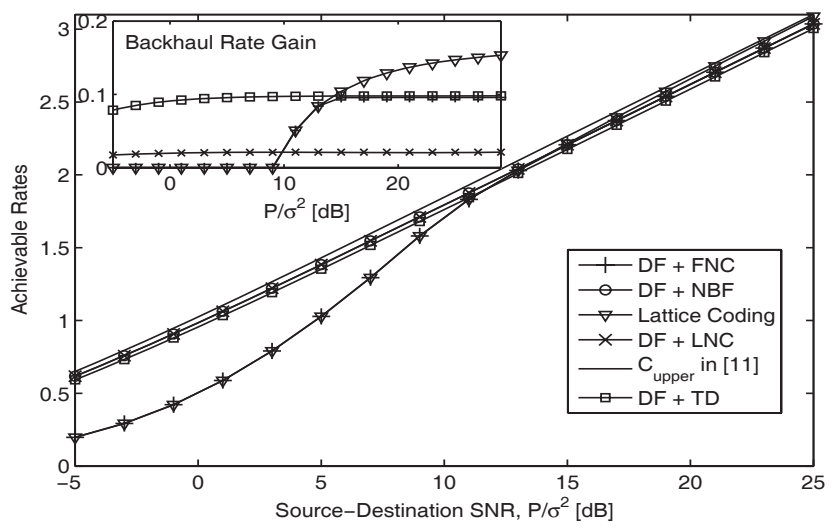

Figure 4. Effects of the source-destination SNR $P / \sigma^{2}$ on the achievable rates with channel gains $\left(a^{2}, b^{2}\right)=(10,10) \mathrm{dB}$.

Significant gain of using backhaul, as shown in the sub-figure, can be obtained for a poor relay-destination link ( small $\left.b^{2}\right)$.

In Fig. 3 we fix $P / \sigma^{2}=5 \mathrm{~dB}$ and $b^{2}=0 \mathrm{~dB}$ instead and vary the source-relay link quality $\left(a^{2}\right)$. Rate gains by using network coding are significant in a large range of $a^{2}$ values. Note that when the source-relay link quality is comparable to the source-destination link, i.e. $a^{2}$ is around $0 \mathrm{~dB}$, the lattice coding strategy is preferred. The gain by using backhual is significant for all schemes for $a^{2}$ larger than $0 \mathrm{~dB}$.

We also look into the asymptotic performance of different strategies under a fixed channel gain $a^{2}=b^{2}=10 \mathrm{~dB}$, as shown in Fig. 4. For low and medium SNR, LNC and NBF are preferred. For high SNR, lattice codes perform the best. Since the relay is connected by high quality channels, i.e. $\left(a^{2}, b^{2}\right)=$ $(10,10) \mathrm{dB}$, the rate gain by using backhaul is not significant.

To illustrate the performance of using lattice coding, we compared it to the NBF at fixed $P / \sigma^{2}=5 \mathrm{~dB}$, shown in Fig. 5 . The relative rate gain of NBF compared with lattice coding is also shown as the contour plot. NBF outperforms lattice coding except when $a^{2}$ is around $0 \mathrm{~dB}$. The performance of $\mathrm{LNC}$, not shown here to make the figure readable, is almost the same as NBF in all the regions.

\section{CONCLUSions}

We have considered a relay-aided two-source two-sink wireless multicast network with a backhual link between the

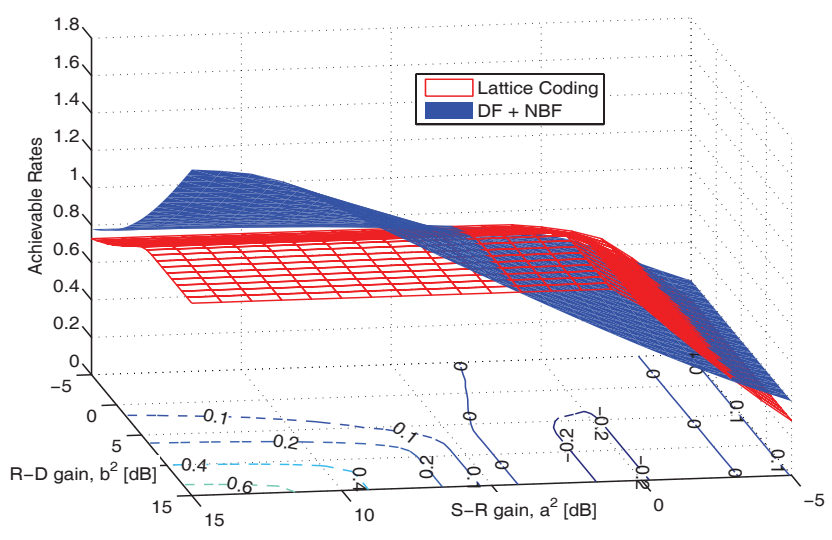

Figure 5. DF+NBF (15) vs. Lattice Coding (12) with $P / \sigma^{2}=5 \mathrm{~dB}$.

source nodes. Different network-coding-based strategies are investigated and compared with a benchmark scheme where relay is time shared by source nodes. Significant rate gains have been demonstrated. In general, linear network coding and NC-based beam-forming strategies give the best performance. Lattice code based strategy is preferred for high SNR or when the source-relay and source-destination channels are comparable. FNC, which only performs modulo-2 addition in the finite field, suffers very limited performance loss in most of the cases. Further, we show significant rate gains compared to the scenarios without backhaul in various channel conditions.

For the future work, we will extend the cooperative strategies in this work to finite-rate backhaul and derive a tighter upper bound than $C_{\text {upper }}$ in [11].

\section{ACKNOWLEDGMENTS}

This work is funded by VINNOVA and Wireless@KTH.

\section{REFERENCES}

[1] T. M. Cover and A. El Gamal, "Capacity theorems for the relay channel," IEEE Trans. Inf. Theory, vol. 25, pp. 572-584, Sep. 1979.

[2] A. Høst-Madsen and J. Zhang, "Capacity bounds and power allocation for wireless relay channels," IEEE Trans. Inf. Theory, vol. 51, pp. 2020 2040, Jun. 2005.

[3] G. Kramer, M. Gastpar and P. Gupta, "Cooperative strategies and capacity theorems for relay networks," IEEE Trans. Inf. Theory, vol. 51, pp. 30373063, Sep. 2005.

[4] Y. Liang and G. Kramer, "Rate regions for relay broadcast channels," IEEE Trans. Inf. Theory, vol. 53, pp. 3517-3535, Oct. 2007.

[5] O. Sahin and E. Erkip, "Achievable rates for the Gaussian interference relay channel", in Proc. of IEEE GLOBECOM, Nov. 2007.

[6] S. Katti, I. Marić, A. J. Goldsmith, D. Katabi, and M. Médard, "Joint relaying and network coding in wireless networks", in Proc. of IEEE ISIT, Jun. 2007.

[7] D. Gündüz, O. Simeone, A. J. Goldsmith, H. V. Poor, and S. Shamai, "Multiple multicasts with the help of a relay," http://arxiv.org/abs/0902.3178v1

[8] C. T. K Ng, N. Jindal, A. J. Goldsmith, and U. Mitra, "Capacity gain from two-transmitter and two-receiver cooperation", IEEE Trans. Inf. Theory, vol. 53, pp. 3822-3827, Oct. 2007.

[9] O. Simeone, D. Gündüz, H. V. Poor, A. J. Goldsmith, and S. Shamai, "Compound multiple-access channels with partial cooperation," IEEE Trans. Inf. Theory, vol. 55, pp. 2425-2441, Jun. 2009.

[10] K. Narayanan, M. P. Wilson, and A. Sprintson, "Joint physical layer coding and network coding for bi-directional relaying," http://arxiv.org/abs/0805.0012v2

[11] B. Wang, J. Zhang, and A. Høst-Madsen, "On the capacity of MIMO relay channels," IEEE Trans. Inf. Theory, vol. 51, pp. 29-43, Jan. 2005.

[12] H. Zhang, N. B. Mehta, and A. F. Molisch, "Asynchronous interference mitigation in cooperative base station systems," IEEE Trans. Wireless Commun., vol. 7, pp. 155-165, Jan. 2008. 